\title{
Thermal Conductivity of Cane Fiberboard (U)
}

by

D. R. Leader

Westinghouse Savannah River Company

Savannah River Site

Aiken, South Carolina 29808

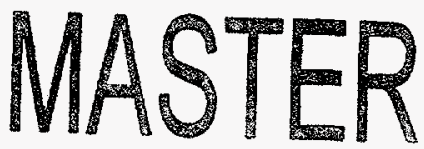

DOE Contract No. DE-AC09-89SR18035

This paper was prepared in connection with work done under the above contract number with the U.S. Department of Energy. By acceptance of this paper, the publisher and/or recipient acknowledges the U.S. Government's right to retain a nonexclusive, royalty-free license in and to any copyright covering this paper, along with the right to reproduce and to authorize others to reproduce all or part of the copyrighted paper.

ESTRIBUTION CF THS DOCLMENT IS UNLIMTRO de 


\section{DISCLAIMER}

This report was prepared as an account of work sponsored by an agency of the United States Government. Neither the United States Government nor any agency thereof, nor any of their employees, makes any warranty, express or implied, or assumes any legal liability or responsibility for the accuracy, completeness, or usefulness of any information, apparatus, product, or process disclosed, or represents that its use would not infringe privately owned rights. Reference herein to any specific commercial product, process, or service by trade name, trademark, manufacturer, or otherwise does not necessarily constitute or imply its endorsement, recommendation, or favoring by the United States Government or any agency thereof. The views and opinions of authors expressed herein do not necessarily state or reflect those of the United States Government or any agency thereof.

This report has been reproduced directly from the best available copy.

Available to DOE and DOE contractors from the Office of Scientific and Technical Information, P. O. Box 62, Oak Ridge, TN 37831; prices available from (423) 576-8401.

Available to the public from the National Technical Information Service. U. S. Department of Commerce, 5285 Port Royal Rd., Springfield, VA 22161 


\section{DISCLAMMER}

Portions of this document may be illegible in electronic image products. Images are produced from the best available original document. 


\section{ASET}

APPLIED SCIENCE \& ENGINEERING TECHNOLOGY

Keywords: Thermal Conductivity

Cane Fiberboard

Retention:

Permanent

\section{THERMAL CONDUCTIVITY OF CANE FIBERBOARD (U)}

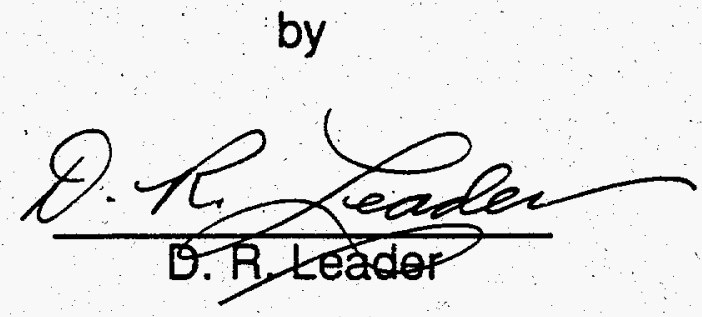

ISSUED: May, 1995

Authorized Derivative Classifier

D. Thame Ravin

SRTC
SAVANNAH RIVER TECHNOLOGY CENTER, AIKEN, SC 29808

Westinghouse Savannah River Company

Prepared for the U. S. Department of Energy under Contract DE-AC09-89SR18035
$6 / 5 / 85$

Date 
DOCUMENT: WSRC-TR-95-0245

IITLE: . THERMAL CONDUCTIVITY OF CANE FIBERBOARD (U)

\section{APPROVALS}

$y_{\text {rex J.Cland }}$

G. T. Chandler, TECHNICAL REVIEWER MATERIALS TECHNOLOGY SECTION

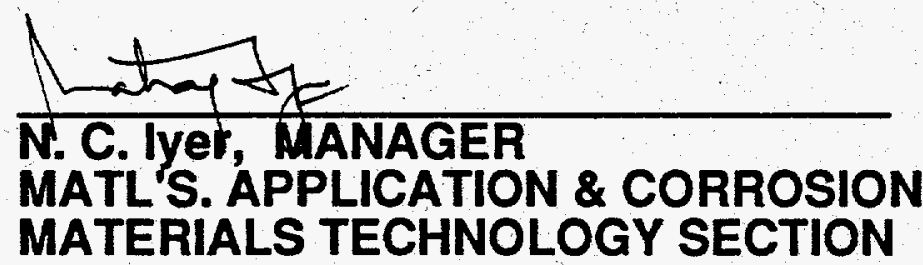

T-Korelos T. L. Capeletti, MANAGER MATERIALS TECHNOLOGY SECTION
DATE: $6 / 6 / 95$

DATE: $6 / 6 / 95$

DATE: $\quad 6 / 15 / 5,5$ 


\section{SUMMARY.}

The thermal conductivity of cane fiberboard was measured in two planes; parallel to the surface and perpendicular to the surface of the manufactured sheet. The information was necessary to better understand the thermal response of a loaded shipping container. The tests demonstrated that the thermal conductivity of cane fiberboard in the plane parallel to the surface of the sheet was nearly twice as great as the conductivity of the same material in a plane perpendicular to the sheet. There was no significant difference in the conductivity in different directions within the plane parallel to the surface, and the presence of glue between layers of fiberboard did not significantly change the conductivity of the assembly. The tests revealed that the thermal conductivity measured in a direction perpendicular to the plane of the surface of a stack of cane fiberboard sheets not bonded together, decreases with an increase in the mean temperature. This was determined to be the result of air gaps between the sheets of fiberboard, and not related to the properties of the material itself.

\section{BACKGROUND}

The thermal conductivity of cane fiberboard samples was measured in the Thermal Measurements Laboratory at the Center for Applied Technology in St. Petersburg, Florida.* The thermal Measurements Lab is a small part of a major commercial testing laboratory that serves the building industry to establish the R-values of building materials.

The lab has the capability of measuring the R-value of assembled doors, windows and walls as well as small sampies of building materials. The thermal conductivity of cane fiberboard samples for these tests was measured in a Dynatech (Cambridge, Mass) "Rapid-K" thermal conductivity instrument. This instrument is designed to measure the thermal transmission properties of a 12" $\times 12$ " sample up to 2 " thick in accordance with ASTM specification C 518-91. The technique uses a heat flux transducer to measure the flow of heat through the center 4" $\times 4$ " section of the sample. The apparatus establishes a steady state unidirectional heat flux through the sample, which is clamped between two parallel plates that are each at constant but different temperatures. The calibration of the instrument is verified periodically per ASTM C 518-91 by measurement of a specimen of known thermal conductivity that is traceable to the National Institute of Standards and Technology (NIST).

* Center For Applied Engineering, Inc. 10301 Ninth Street North, St. Petersburg FL 33716, Phone (813) 576-4171. 
Cane fiberboard is used in shipping containers for the weapons complex both as thermal insulator and energy absorber to cushion the payload under impact conditions. Disks that are slightly smaller than the inside diameter (ID) of a stainless steel drum are cut from $1 / 2^{\prime \prime}$ thick $4^{\prime} \times 8^{\prime}$ sheets of cane fiberboard and stacked in the drum to completely fill it. A cylindrical hole is cut in the center of the stack for placement of the containment vessel that is to be shipped. In the older shipping containers, the stack of

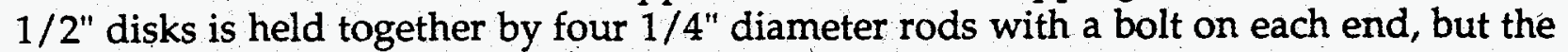
latest design requires the disks to be glued together.

Cane fiberboard has been used by the building construction industry for many years as sheathing on the outside walls of residential and commercial buildings. It is manufactured from the crushed stalks of the sugar cane plant as well as some cellulose from recycled newsprint paper. Because the long cane fibers lie primarily in a plane parallel to the surface of the board, and because fibers are likely to transfer more heat along their axis than across them, it is reasonable to assume that cane fiberboard would have a higher thermal conductivity parallel to the surface than perpendicular to the surface. Although thermal conductivity perpendicular to the plane surface of the $1 / 2$ " thick sheet is well documented, no published information is available for the thermal conductivity parallel to the plane surface of the sheet.

Knowledge of thermal conductivity in both planes is necessary to understand the performance and thermal response of a loaded shipping package, because most of the heat is transferred through the fiberboard in a plane parallel to the surface of the sheets.

\section{SAMPLE DESCRIPTION}

The thermal conductivity of individual sheets of fiberboard as well as five different assemblies was measured in these tests. A description of each sample follows (The designation in brackets is the sample designation used in the test report from the Center for Applied Engineering]:

Sample Assembly \#1 (L-flat\} - Four $12^{\prime \prime} \times 12^{\prime \prime} \times 1 / 2^{\prime \prime}$ sheets of cane fiberboard held together by water-base carpenters wood glue that was applied to the outer 1-inch edge of each surface. The purpose of the glue was to hold the four pieces together without having any influence on the thermal conductivity of the center 4 " $\times 4$ " area where the heat flux was measured. This sample simulates fiberboard in the "old" packages, in which the sheets of fiberboard are not bonded together.

Sample Assembly \#2 \{L - A "picture frame" was made by cutting a 11 " $\times 11$ " opening in the center of a stack of four $12^{\prime \prime} \times 12^{\prime \prime}$ sheets of $1 / 2^{\prime \prime}$. thick cane fiberboard that have been glued together. Twenty two strips of cane fiberboard 2 " wide, 11 " long and 1/2" thick were stacked into the opening of the picture frame. Each of the strips was cut with the 11 inch dimension parallel to the 8-foot length of the original sheet of cane fiberboard. The strips were held in the picture frame by friction as the result of a close fit - no glue was used to hold them in place. This sample was used to determine the thermal 
conductivity of fiberboard in a plane parallel to the surface. Both 12 " $\times 12^{\prime \prime}$ surfaces of this sample were smoothed by rubbing the sample across a plane surface coated with abrasive paper. This was done to assure good contact with the surface of the plates in the test instrument.

Sample Assembly \#3 [W] - This sample is identical to sample \#2 in every respect except that the strips were cut with the 11-inch dimensions parallel to $t$ he 4-foot dimension of the original sheet of cane fiberboard. This sample was used to determine if there was any difference in thermal conductivity that was related to the orientation of the fiberboard. Both $12 " \times 12^{\prime \prime}$ surfaces of this sample were smoothed by rubbing the sample across a plane surface coated with abrasive paper. This was done to assure good contact with the surface of the plates in the test instrument.

Sample Assembly \#4 (L-glue\} - This sample is identical to sample \#2 in every respect except that the $2^{\prime \prime} \times 11^{\prime \prime}$ surfaces of each strip were coated with water based carpenters wood glue before assembly. This sample was used to determine if the glue had any influence on thermal conductivity of fiberboard measured in a plane parallel to the surface. Both $12^{\prime \prime} \times 12^{\prime \prime}$ surfaces of this sample were smoothed by rubbing the sample across a plane surface coated with abrasive paper. This was done to assure good contact with the surface of the plates in the test instrument.

Sample Assembly \#5 (Series III)- This sample was similar to sample number 1, except that the surface area of adjacent sheets was coated with glue to form an essentially solid sample. This sample was used to determine the effect of glued assembly on conductivity measured perpendicular to the surface of the fiberboard.

In addition to the five assemblies described above, four individual 12 " $\times 12$ " samples of $1 / 2^{\prime \prime}$ thick cane fiberboard were used in the testing process. The individual sheets were never fastened together in any way, but the thermal conductivity was measured on one sheet, then a stack of two sheets, then a stack of three sheets and finally a stack of four sheets. These samples were used in the "SERIES II" tests and were designated 1-flat, 2flat, 3-flat and 4-flat.\} Sample assemblies \#1, \#2, \#3 and \#4 and the four individual samples were made from the same $4^{\prime} \times 8^{\prime}$ sheet of cane fiberboard sheathing, but sample assembly $\# 5$ was made from a different sheet.

\section{TEST DESCRIPTION}

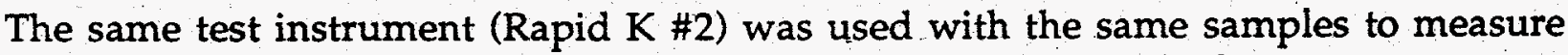
thermal conductivity at two different mean temperatures. The high temperature tests were run at an average sample temperature of $180^{\circ} \mathrm{F}$ and an average temperature difference between the hot and cold surface of $40^{\circ} \mathrm{F}$. Low temperature tests were done on the same samples at an average sample temperature of $75^{\circ} \mathrm{F}$ and an average temperature difference between the hot and cold surface of $40^{\circ} \mathrm{F}$. Sample Assembly \#4 was not run at the high temperature. The calibration of the machine was verified before and after each series of tests by measuring the conductivity of a standard sample of known conductivity verified by the NIST. 
In each test, the sample is clamped between the two temperature controlled plates in the thermal conductivity measurement apparatus. The energy required to maintain the two surface temperatures at the specified values is controlled by a computer. After equilibrium conditions are established and maintained for a predetermined time, the computer automatically calculates the thermal conductivity of the sample. The minimum time required for a sample to reach equilibrium in the test equipment ranges between one and three hours, but the test can be continued for as long as desired.

Photograph \#1 shows the test instrument with sample \#3 being held in front of the open door. After the sample is placed into the instrument, a lever on the right side (not seen in the photograph) raises the lower surface and applies a spring loaded force on the sample so it is in contact with both the lower and upper surface of the instrument. The front door, which is insulated, is then closed and latched. Only the edge of the door and the latch are visible in the photograph. The dial indicator on the top of the instrument indicates the thickness of the sample being tested.

Photograph \#2 is the display for the computer that controls six different instruments in the laboratory. Sample \#1 [L-flat\} had been in instrument RX 2 (center top in the display) for 115 minutes when this photograph was made.

There were three series of tests done on the samples: Series I tests were run on samples $\# 1, \# 2$, \#3 at two different temperatures and at the low temperature only on sample \#4. The data from these tests are summarized in Table I.

Series II tests were done with four individual $12^{\prime \prime} \times 12^{\prime \prime}$ samples of fiberboard. Thermal conductivity was measured on each individual sample and on stacks of 2,3 and 4 samples at both the $75^{\circ} \mathrm{F}$ and the $180^{\circ} \mathrm{F}$ mean temperature. Results of series II tests are summarized in Table II.

Series III thermal conductivity tests were all done on sample number 5 . Six or seven different tests at each temperature were done on the same sample of four sheets that were glued together to form a solid assembly. Results of series III tests are summarized in Table III.

\section{RESULTS AND DISCUSSION}

The test results from the series I tests are summarized in Table I. The results confirm the hypothesis that the thermal conductivity in the plane parallel to the surface of the sheet is greater than the conductivity perpendicular to the surface. This is a reasonable result because the long hollow cane fibers which are inclined to conduct heat preferentially along their length are randomly oriented with their axis in a plane more or less parallel to the surface.

Samples \#2 and \#3 differed only in the orientation of the sample relative to the length of the original sheet of material. Although the thermal conductivity is slightly higher for 
the sample cut in the direction parallel to the 4 -foot dimension of the original sheet of fiberboard for the low temperature test, the opposite is true at the high temperature. The difference appears to be within the range of experimental error. Again, this is a reasonable result because there is no apparent alignment of the long cane fibers in any one direction.

Samples \#2 and \#4 differed only in the fact that the 22 individual pieces in the sample were glued together in sample \#4. Although the conductivity for sample \#4 is slightly higher, the difference does not appear to be significant. The glue line is very thin relative to the sample, and even if the glue had a slightly different coefficient of heat transfer than the fiberboard, it would not be likely to make a major change in the measured coefficient of heat transfer for the assembly.

One apparently anomalous result of these tests was that the thermal conductivity of sample 1 decreased at the higher mean temperature. Because this is inconsistent with the expected behavior of cane fiberboard, the second series of tests was run. Sample \#1 was made from four 12" $\times 12$ " sheets that were glued together only at the edges and the coefficient of heat transfer is measured at the 4 " square in the center of the sample.

A second series of tests was done to determine the reason for the apparently anomalous test results from sample \#1. The tests results from the series II tests are summarized in table II. In these tests, the thermal conductivity of four individual boards was first measured individually and then in stacks two, three and four sheets thick. These tests confirmed the behavior of sample \#1 in the series I tests. The thermal conductivity of a stack of sheets decreased with an increase in the mean temperature. At this point it was postulated that the mechanism for this unusual behavior was the result of the air gap between adjacent sheets in a stack of cane fiberboard that is not glued together.

Series III tests were run to verify the hypothesis that the air gap between sheets on sample \#1 and the samples in the series II tests caused the thermal conductivity to decrease with an increase in temperature. The tests results from the series III tests are included at the bottom of table I. In this test, four sheets of fiberboard were glued together to form a solid sample 12" $\times 12^{\prime \prime} \times 2$ ". Thermal conductivity was measured at both the high and low mean temperature in the direction perpendicular to the face of the sheets. The tests were repeated at least six times and all test results were within the range expected for an experiment of this type. The thermal conductivity of the sample increased, as expected, at the higher temperature. These results verify the hypothesis that the decrease in measured thermal conductivity at higher temperatures for a stack of fiberboard that is not bonded together is the result of the air gap between the individual sheets of fiberboard, and is not related to some peculiar property of the material itself.

A copy of the test report from the Center for Applied Engineering is attached to this memo along with a copy of all of the original test data. 


\section{CONCLUSIONS}

The thermal conductivity of cane fiberboard in a direction parallel to the surface is about twice the value of conductivity through the sheet. There is no significant difference in conductivity measured in different directions parallel to the surface based on the results of tests in two directions. The use of wood glue to bond multiple sheets together does not influence the conductivity in a direction parallel to the surface of the sheets, but the presence of an air gap between layers that are not bonded together results in decreased thermal conductivity at higher temperatures for a stack of unbonded sheets of fiberboard. All of the results appear reasonable and in agreement with the expected behavior of this material. 
TABLE I

\begin{tabular}{|c|c|c|c|c|}
\hline $\begin{array}{l}\text { SAMPLE } \\
\text { ID }\end{array}$ & $\begin{array}{l}\text { MEAN } \\
\text { TEMP. }{ }^{\circ} \mathrm{F}\end{array}$ & $\begin{array}{l}\text { Apparent Thermal } \\
\text { Conductivity } \\
\left.\text { (Btu in } / \mathrm{h} \mathrm{ft}^{2}{ }^{\circ} \mathrm{F}\right)\end{array}$ & $\begin{array}{l}\text { Direction of } \\
\text { Heat Flow } \\
\text { Relative to } \\
\text { the Surface } \\
\text { of the sheet }\end{array}$ & $\begin{array}{c}\text { Sheets } \\
\text { Glued } \\
\text { Together } \\
?\end{array}$ \\
\hline 1 (L-Flat $\}$ & 74.7 & $0.420(1)$ & Perpendicular & No \\
\hline 1 (L-Flat\} & 179.9 & 0.290 (1) & Perpendicular & No \\
\hline $2\{\mathrm{~L}\}$ & 75.0 & $0.762(2)$ & Parallel & No \\
\hline $2\{\mathrm{~L}\}$ & 179.9 & 0.755 (1) & Parallel & No \\
\hline $3\{W\}$ & 75.1 & $0.803(2)$ & Parallel & No \\
\hline $3\{W\}$ & 180.3 & 0.727 (1) & Parallel & No \\
\hline 4 \{L-Glue\} & 75.0 & 0.797 (2) & Parallel & Yes \\
\hline 4 \{L-Glue\} & 180.0 & Not measured & & \\
\hline 5 \{SERIES III & 74.9 & 0.398 (1) & Perpendicular & Yes \\
\hline 5 \{SERIES III\} & 180.0 & $0.440(1)$ & Perpendicular & Yes \\
\hline
\end{tabular}

Notes:

(1) Average of two or more test results.

(2) Single test result. 
TABLE II

\begin{tabular}{|c|c|c|c|}
\hline Sample ID & $\begin{array}{c}\text { Mean } \\
\text { Temperature }\left({ }^{\circ} \mathrm{F}\right)\end{array}$ & Thickness (inçh) & 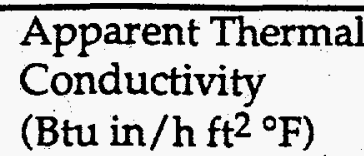 \\
\hline \multicolumn{4}{|c|}{ Series II Tests } \\
\hline$\longdiv { 1 - \text { flat } }$ & $\overline{74.6}$ & 0.523 & 0.387 \\
\hline 2-flat & 74.8 & 0.515 & 0.391 \\
\hline 1,2 -flat & 74.9 & 1.024 & 0.411 \\
\hline 3-flat & 75.1 & 0.511 & 0.391 \\
\hline 1,2,3-flat & 74.8 & 1.547 & 0.426 \\
\hline 4-flat & 74.9 & 0.527 & 0.381 \\
\hline 1,2,3,4-flat & 75.4 & 2.058 & 0.415 \\
\hline 1-flat & 180.2 & 0.527 & 0.470 \\
\hline 2-flat & 180.1 & 0.529 & 0.460 \\
\hline 1,2-flat & 179.9 & 1.041 & 0.420 \\
\hline 3-flat & 180.1 & 0.529 & 0.450 \\
\hline 1,2,3-flat. & 179.9 & 1.554 & 0.285 \\
\hline 4-flat & 180.3 & 0.528 & 0.449 \\
\hline 1,2,3,4-flat & 180.1 & 2.064 & 0.165 \\
\hline
\end{tabular}




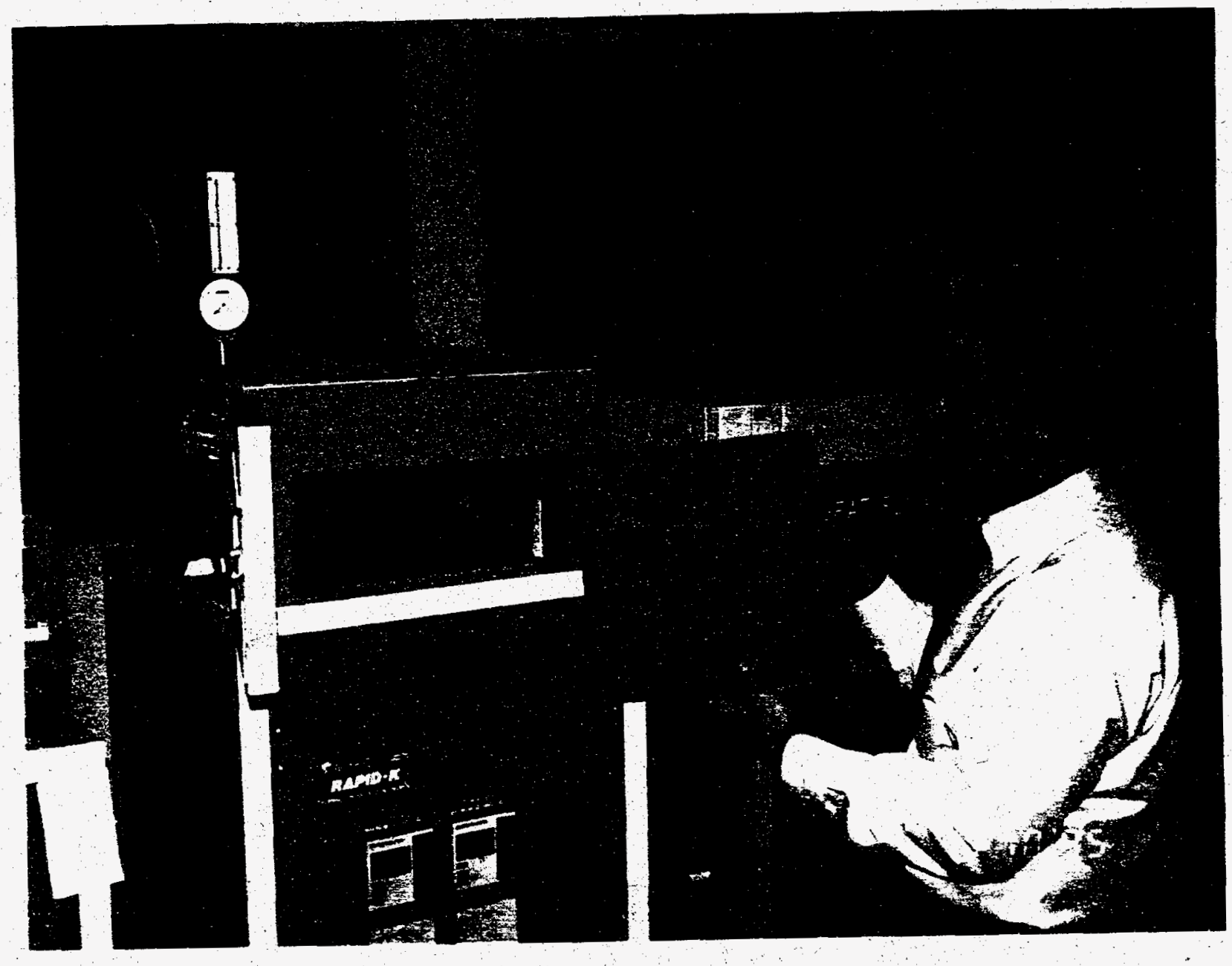

Photograph \#1: Conductivity Measurement Instrument 


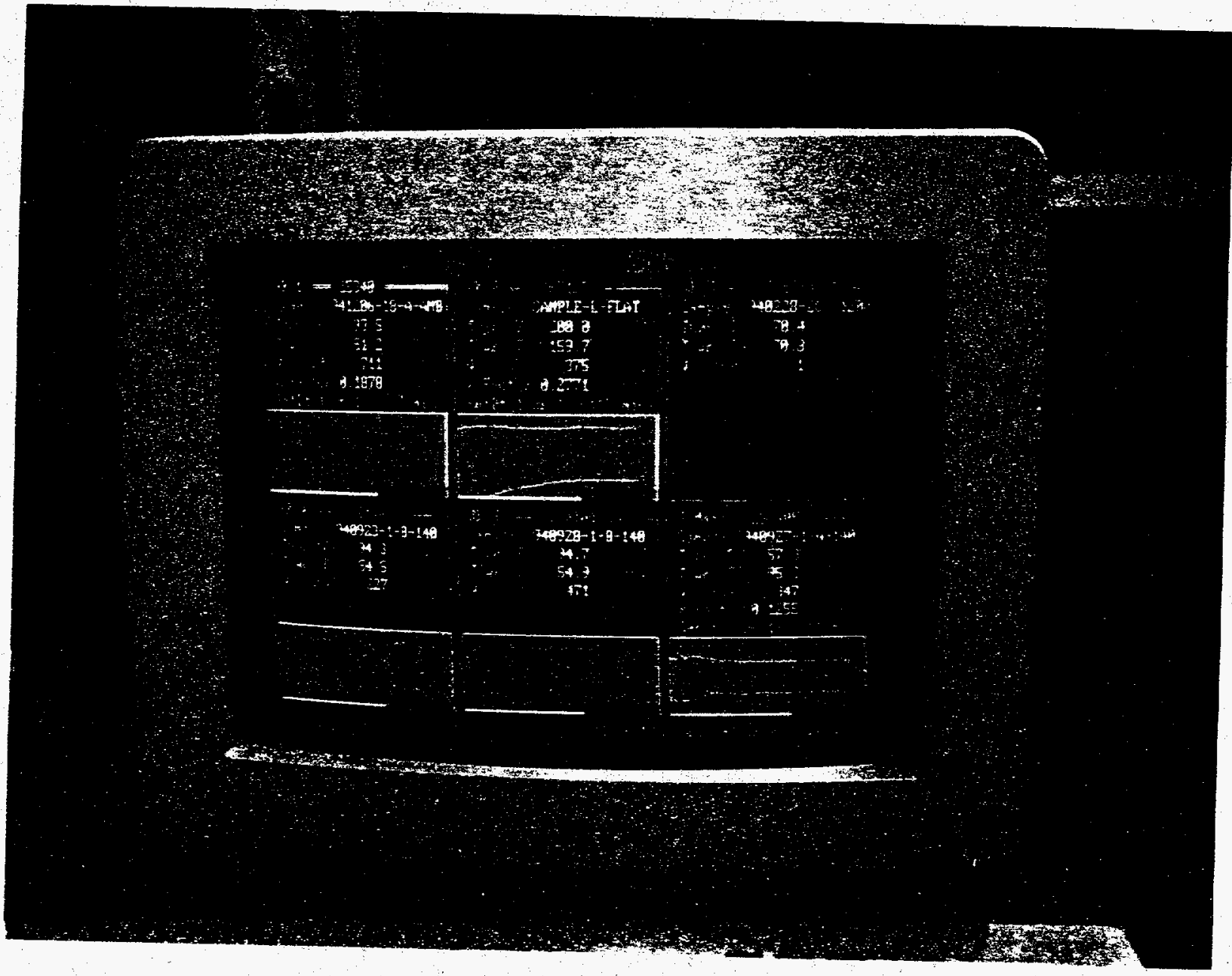

Photograph \#2: Computer Display 


\section{(1) Center For Applied \\ SM Materials Testing Services}

February 28, 1995

To: Mr. Douglas R. Leader

Westinghouse Savannah River Company

P. O. Box 616

Bldg. 773-A, Room D-1142

Aiken, SC 29802-0616

Subject: ASTM C518 Tests on Cane Fiberboard

\section{SERIES I TESTS}

Series $I$ tests were conducted on samples identified by the client as $L$-flat, $L$-glue, $L$ and $W$. Tests at mean temperatures of $180^{\circ} \mathrm{F}$ and $75^{\circ} \mathrm{F}$ were conducted on all samples except L-glue which was tested at $75^{\circ} \mathrm{F}$ only. Test results are summarized on the attached table. The L-flat data are questionable as the apparent thermal conductivity at $180^{\circ} \mathrm{F}$ was lower than at $75^{\circ} \mathrm{F}$. This is inconsistent with typical thermal data.

\section{SERIES II TESTS}

Series II tests were conducted on samples identified as 1-flat, 2-flat, 3-flat and 4-flat. After testing individual samples, samples were stacked and identified as 1,2-flat; 1,2,3-flat; and 1,2,3,4-flat. Tests were conducted on all samples at both $180^{\circ} \mathrm{F}$ and $75^{\circ} \mathrm{F}$ mean temperature, a total of 14 tests. Densities of the 4 samples were:

$\begin{array}{ll}\text { 1-flat } & 15.3 \mathrm{lb} / \mathrm{ft}^{3} \\ \text { 2-flat } & 15.9 \mathrm{lb} / \mathrm{ft}^{3} \\ \text { 3-flat } & 15.7 \mathrm{lb} / \mathrm{ft}^{3} \\ \text { 4-flat } & 15.3 \mathrm{lb} / \mathrm{ft}^{3}\end{array}$

Test results are summarized on the attached Table. The data obtained at $75^{\circ} \mathrm{F}$ mean temperature appear to be consistent with the overall average thermal conductivity obtained at $75^{\circ} \mathrm{F}$ from Series III. The $180^{\circ} \mathrm{F}$ data are questionable as the thermal conductivity should not decrease as additional samples are stacked, i.e., 1,2,3-flat and 1,2,3,4-flat.

This zepon is tor the information of the client. It may $\$ 0$ useo in its entipeny for the purpose of securing product accoptance from duly constituted approva! authorities: however, this reper or the naris of Center For Appied Engineering; Inc. shall not be used in publicily or advertising.

Accredited by the National Institute of Standards and Technology, National Voluntary I.aboratory Accreditation Program for selected test methods for Acoustical Test Services and Thermal insulation Materiais.

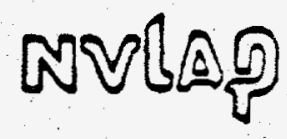

ACOUSTICAL, FIRE, PHYSICAL AND THERMAL MEASUREMENTS LABORATORIES 


\section{Engineering,Inc.

Mr. Douglas R. Leader - Continued

Westinghouse Savannah River Company

Page 2

February 28, 1995

\section{SERIES III TESTS}

Series III tests were conducted on a stack of four (4) nominal 0.5 -inch thick fiberboard samples. The 4 samples were adhered with Carpenter's glue and allowed to dry overnight with heavy weights on top. The stack was placed in an oven and dried for approximately 24 hours at $215^{\circ} \mathrm{F}$. The initial weight was 1345.9 grams; after drying the weight was 1178.5 grams. The stack was placed in a desiccator over the weekend and reweighed just prior to testing, 1182.0 grams. After testing at $180^{\circ} \mathrm{F}$ all day, the stack was reweighed, 1182.2 grams. The density of the sample was $15.7 \mathrm{lb} / \mathrm{ft}^{3}$.

For both mean temperature tests, the stack was left in the heat flow meter all day with ASTM C518 acceptable data taken throughout the day. As can be seen in the attached Table, the data are extremely constant for the 6 or 7 readings. These data are also as would be expected, the thermal conductivity at $180^{\circ} \mathrm{F}$ is approximately 11 percent higher than at $75^{\circ} \mathrm{F}$.

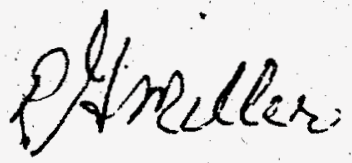

R. G. MILLER

Manager

Materials Testing Services

RGM:dds

encl. 
Center For Applied

Engineering, lnc.

Materials Testing Services

\section{SUMMARY OF FIBERBOARD TEST RESULTS}

\begin{tabular}{|c|c|c|c|}
\hline Sample ID & $\begin{array}{l}\text { Mean } \\
\text { Temperature }\left({ }^{\circ} \mathrm{F}\right)\end{array}$ & Thickness (inch) & $\begin{array}{l}\text { Apparent Thermal } \\
\text { Conductivity } \\
\left(\text { Btu in } / \mathrm{h} \mathrm{ft}^{2} \circ \mathrm{F}\right)\end{array}$ \\
\hline \multicolumn{4}{|c|}{ Series I Tests } \\
\hline L-Flat & 74.3 & 2.083 & 0.432 \\
\hline & 75.1 & 2.075 & 0.407 \\
\hline \multirow[t]{3}{*}{ Average } & 74.7 & 2.079 & 0.420 \\
\hline & 179.8 & 2.102 & 0.280 \\
\hline & 180.0 & 2.081 & 0.299 \\
\hline Average & 179.9 & 2.092 & 0.290 \\
\hline L-Glue & 75.0 & 2.012 & 0.797 \\
\hline \multirow[t]{3}{*}{$L$} & 75.0 & 2.008 & 0.762 \\
\hline & 179.9 & 2.020 & 0.772 \\
\hline & 179.9 & 2.008 & 0.737 \\
\hline Average & 179.9 & 2.014 & 0.755 \\
\hline \multirow[b]{5}{*}{ Average } & 75.1 & 2.017 & 0.803 \\
\hline & 180.5 & 2.029 & 0.795 \\
\hline & 180.2 & 2.021 & 0.704 \\
\hline & 180.2 & 2.021 & 0.682 \\
\hline & 180.3 & 2.024 & 0.727 \\
\hline
\end{tabular}

This repon is for the information of the client. $\mathrm{k}$ may be used in its entirety for the purpose of securing product acceptance from 
Center For Applied

Engineering, Inc.

Materials Testing Services

\section{SUMMARY OF FIBERBOARD TEST RESULTS}

\begin{tabular}{|c|c|c|c|}
\hline Sample ID & $\begin{array}{l}\text { Mean } \\
\text { Temperature }\left({ }^{\circ} \mathrm{F}\right)\end{array}$ & Thickness (inch) & $\begin{array}{l}\text { Apparent Thermal } \\
\text { Conductivity } \\
\left(\text { Btu in } / \mathrm{h} \mathrm{ft}^{2}{ }^{\circ} \mathrm{F}\right)\end{array}$ \\
\hline \multicolumn{4}{|c|}{ Series II Tests } \\
\hline 1-flat & 74.6 & 0.523 & 0.387 \\
\hline 2-flat & 74.8 & 0.515 & 0.391 \\
\hline 1,2 -flat & 74.9 & 1.024 & 0.411 \\
\hline 3-flat & 75.1 & 0.511 & 0.391 \\
\hline $1,2,3$-flat & 74.8 & 1.547 & 0.426 \\
\hline 4-flat & 74.9 & 0.527 & 0.381 \\
\hline $1,2,3,4-$ flat & 75.4 & 2.058 & 0.415 \\
\hline 1 -flat & 180.2 & 0.527 & 0.470 \\
\hline 2 -flat & 180.1 & 0.529 & 0.460 \\
\hline 1,2 -flat & 179.9 & 1.041 & 0.420 \\
\hline 3-flat & 180.1 & 0.529 & 0.450 \\
\hline $1,2,3$-flat & 179.9 & 1.554 & 0.285 \\
\hline 4-flat & 180.3 & 0.528 & 0.449 \\
\hline $1,2,3,4$-flat & 180.1 & 2.064 & 0.165 \\
\hline
\end{tabular}

$\mathrm{n}$ is for the infurmation of the client. ht may be used in its entirey for the purpose of securing product acceptance from 


\section{SUMMARY OF FIBERBOARD TEST RESULTS}

\begin{tabular}{|c|c|c|c|}
\hline Sample ID & $\begin{array}{l}\text { Mean } \\
\text { Temperature }\left({ }^{\circ} \mathrm{F}\right)\end{array}$ & Thickness (inch) & $\begin{array}{l}\text { Apparent Thermal } \\
\text { Conductivity } \\
\left.\text { (Btu in/h } \mathrm{ft}^{2} \circ \mathrm{F}\right)\end{array}$ \\
\hline \multicolumn{4}{|c|}{ Series III. Tests } \\
\hline \multicolumn{4}{|c|}{ 4, Nominal 0.5-inch Samples of Fiberboard Glued with Carpenter's Glue } \\
\hline 1 & 180.0 & 2.055 & 0.430 \\
\hline 2 & 179.9 & 2.055 & 0.442 \\
\hline 3 & 180.0 & 2.055 & 0.444 \\
\hline 4 & 180.0 & 2.055 & 0.443 \\
\hline 5 & 180.0 & 2.055 & 0.441 \\
\hline 6 & 179.9 & 2.055 & 0.441 \\
\hline Average & 180.0 & 2.055 & 0.440 \\
\hline 1 & 74.9 & 2.038 & 0.395 \\
\hline 2 & 74.9 & 2.038 & 0.400 \\
\hline 3 & 74.9 & 2.038 & 0.399 \\
\hline 4 & 74.9 & 2.038 & 0.398 \\
\hline 5 & 74.9 & 2.038 & 0.397 \\
\hline 6 & 74.9 & 2.038 & 0.397 \\
\hline 7 & 74.9 & .2 .038 & 0.398 \\
\hline Average & 74.9 & 2.038 & 0.398 \\
\hline
\end{tabular}


Req No: 950041

Date: $01-11-1995$

Steady-State Thermal Trarsmission Properties

by Veans of the Heat Flow Neter per ASTM C $51 \mathrm{~s}$

Project no: 057165

Runi ID: 257165

Sample Description: SAMPLE-L-FLAT-AMD.

specimen orientation: Horizontal Heat Meter Location: Cold Surface Instrument Used: Rapid-k

Duration of Measurement:

iverage Heat Flux

Average Hot Plate Temperature Th

Average cold Plate Temperature Tc

Average Temperature Difference

Average Sample Temperature

Average Thermal Resistance $R$

Average Thermal Conductance $C$
181 minutes.

$=8.57 \mathrm{Btu} /(\mathrm{h} \mathrm{sq}-\mathrm{ft})$

$=94.9$ deg $F$

$=53.6 \operatorname{deg} \mathrm{F}$

$=41.3 \operatorname{deg} F$

$=-4.3$ deg $F$

$=4.82 \mathrm{~h} \mathrm{sq}-\mathrm{ft} \mathrm{F} / \mathrm{Btu}$

$=0.207$ Btu/(h sq-ft $F)$

Calculations Based on Thickness. Measured in Apparatus

Thickness

Average Temperature Gradient

Apparent Thermal Conductivity
$=2.083$ inch

$=19.83 \mathrm{deg}$ F/inch

$=0.432 \mathrm{Btu}-\mathrm{in} /(\mathrm{h} \mathrm{sq}-\mathrm{ft} \mathrm{F})$

operator:

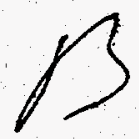

Comments 
Req No: 950041

Date: $01-11-1995$

Steady-State Thermal Transmission Properties

by Veans of the Heat Flow Hoter per ASTM $C 518$

Project io: $257165 \quad$ Run ID: 257165

Sample Desciption: SAMPLE-L-FLAT-AME

specimen orientation: Horizontal Heat Meter Location: Cold Surface Instrument Used: Rafid-k $\# 2$

Duration of Veasurement:

Aterage Heat Flux

Average Hot Plate Temperature Th

Average Cold Plate Tenperature I

Average Temperature Difference

Averaje Sample Temperature

Arerage Thermal Resistance $R$

Average Thermal Conductance $C$
81 minutes

$=7.30 \mathrm{Btu} /(\mathrm{h} \mathrm{sq}-\mathrm{ft})$

$=95.0 \mathrm{deg} F$

$=55.2$ deg $F$

$=39.8 \operatorname{deg} F$

$=-5.1$ deg $F$

$=5.09$ h sq-ft F/Btu

$=0.196 \mathrm{Btu} /(\mathrm{h} \cdot \mathrm{sq}-\mathrm{ft} \mathrm{F})$

Calculations Based on Thickness Measured in Apparatus

Thickness

Average Temperature Gradient

Apparent Thermal Conductivity $\mathrm{k}$
$=2.075 \mathrm{inch}$

$=19.16$ deg F/inch

$=0.407$ Btu-in/(h sq-ft F)

operator:

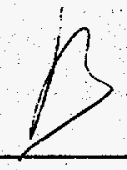

Comments 
Req No: $95004 i$

Date: $\quad 01-10-1995$

Steady-State Thermal Transmission Froperties by leans of the Heat Flow Meter per isTM 6518

Project No: 257165

Run ID: 257:165

Sample Description: SAMPLE-L-FLAT

Specimen Orientation: Horizontal Heat Meter Location: Cold Surface Instrument Used: Rapid-k $\# 2$

Duration of Measurement:

125 minutes

Sterage Heat Flux

$=5.36 \mathrm{Btu} /(\mathrm{h} \mathrm{sq}-\mathrm{ft})$

Average Hot Plate Temperature Th

$=200.0$ deg $F$

Average Cold Plate Temperature Tc

$=159.7$ deg $F$

Average Temperature Difference

$=40.3 \operatorname{deg} F$

Average Sampie Temperature

$=179.8$ deg $F$

Average Thermal Resistance $R$

$=7.51 \mathrm{~h} s q-\mathrm{ft} F / B t u$

Average Thermal Conductance $C$

$=0.133 \mathrm{Btu} /(\mathrm{h} \mathrm{sq}-\mathrm{ft} F)$

Calculations Based on Thickness Measured in Apparatus

Thickness

Average Temperature Gradient

Apparent Thermal Conductivity $k$
$=2.102$ inch

$=19.17 \mathrm{deg} F /$ inch

$=0.280 \mathrm{Btu}-\mathrm{in} /(\mathrm{h}$ sq-ft F)

Operator:

Comments

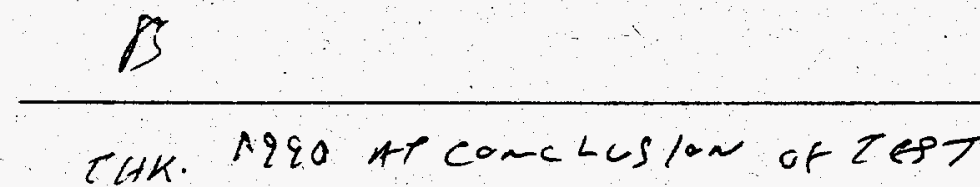


Req No: 950041

Date: $\quad 01-12-1995$

Steady-State Thermal Transmissior Froperties

by Heans of the Heat flow Meter per ASTM C 518

Dioject No: 257165

Run ID: 257165

sample Description: SAMPLE-I-FLAT

Specimen Orientation: Horizontal Heat Meter Location: Cold Surface Instrument Used: Rapid-k $\# 2$

Duration of Measursment:

Arerage Heat Flus

Arerage Hot Plate Temperature Th

Average Cold Plate Temperature To

Average Temperature Difference

Averge Sample Temperature

Averago Thormal Resistance $R$

Aierage Thermal Conductance $C$
135 minutes

$=5.37$ Btu/(h sq-ft)

$=200.4 \operatorname{deg} \mathrm{F}$

$=159.5 \operatorname{deg} F$

$=40.9 \operatorname{deg} F$

$=180.0$ deg $F$

$=6.96 \mathrm{~h} \mathrm{sq}-\mathrm{ft} \mathrm{F/Btu}$

$=0.144 \mathrm{Btu} /(\mathrm{h} \mathrm{sq}-\mathrm{ft} F)$

Calculations Based on Thickness Masured in Apparatus

Thickness

Average Temperature Gradient

Apparent Thermal Conductivity $\mathrm{k}$
$=2.081 \mathrm{inch}$

$=19.64 \mathrm{deg} F /$ inch

$=0.299 \mathrm{Btu}-\mathrm{in} /(\mathrm{h} \mathrm{sq}-\mathrm{ft} F)$

operator:

Comments 
Req No: 950041

Date: 01-11-1995

Steady-State Thermal Transmission Properties

by Means of the Heat Flow Meter per ASTM C 518

Project No: 257165

Run ID: $25: 165$

Sample Description: SAMPLE-L-GLUE-AMB

Specimen Orientation: Horizontal Heat Meter Location: Cold Surface Instrument Used: Rapid-k $\# 2$

Duration of Measurement:

Average Heat Flux

Average Hot Plate Temperature Th

Average Cold Plate Temperature TC

Average Temperature Difference

Average Sampla Temperature

$\therefore$ Forage Thermal Resistance $R$

Average Thermal Conductance $C$
66 minutes

$=15.66 \mathrm{Btu} /(\mathrm{h} \mathrm{sq}-\mathrm{ft})$

$=94.8 \operatorname{deg} F$

$=55.3$ deg $F$

$=39.5 \mathrm{deg} F$

$=75.0$ deg $F$

$=2.52 \mathrm{~h} \mathrm{sq}-\mathrm{ft} F /$ Btu

$=0.396 \mathrm{Btu} /(\mathrm{h} \mathrm{sq}-\mathrm{ftF})$

Calculations Based on Thickness Measured in Apparatus

Thickness

Average Temperature Gradient

Apparent Thermal Conductivity $k$
$=2.012 \mathrm{inch}$

$=19.64 \mathrm{deg} / \mathrm{f} / \mathrm{inch}$

$=0.797 \mathrm{Btu}-\mathrm{in} /(\mathrm{h} \mathrm{sq}-\mathrm{ft} F)$

Operator:

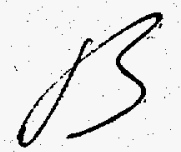

Comments 
Req No: 950041

Date: 01-11-1995

Steady-state Thermal Transmission Properties

by Means of the Heat Flow Meter per ASTM C 518

Froject No: 257165

Run ID: $25 ? 165$

Sample Description: SAMPLE L

Specimen orientation: Horizontal Heat Meter Location: Cold Surface Instrument Used: Rapid-k $\# 2$

Duration of Measurement:

Average Heat Flux

Average Hot Plate Temperature Th

Average cold Plate Temperature TC

Average Temperature Difference

Average Sample Temperature

Average Thermal Resistance $R$

Average Thermal Conductance $C$
76 minutes

$=15.11 \mathrm{Btu} /(\mathrm{h} s q-f t)$

$=94.9 \operatorname{deg} F$

$=55.1$ deg $F$

$=39.8 \cdot \operatorname{deg} F$

$=75.0 \quad \operatorname{deg} F$

$=2.64 \mathrm{~h} \mathrm{sq}-\mathrm{ft} F / B t u$

$=0.379$ Btu/(h sq-ft F)

Calculations Based on Thickness Measured in Apparatus

Thickness

Average Temperature Gradient

Apparent Thermal Conductivity $\mathrm{k}$
$=2.00 \mathrm{~s}$ inch

$=19.83$ deg F/inch

$=0.762 \mathrm{Btu}-\mathrm{in} /(\mathrm{h} \mathrm{sq}-\mathrm{ft} \mathrm{F})$

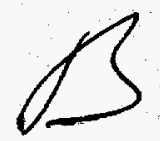

operator:

Comments 
Req No: 950041

Date: $01-10-1995$

Steady-State Thermal Transmission Properties.

by Veans of the Heat Flow Meter per ASTM C $51 \mathrm{~S}$

Project No: 257165

Run ID: 257165

Sample Deseription: SAMPLE-L

Se $=1416$

Specimen orientation: Horizontal Heat Meter Location: Cold Surface Instrument Used: $\quad$ Rapid-k $\# 2$

Duration of Measurement:

Average Heat Flux

Average Hot Plate Temperature Th

Average Cold Plate Temperature TC

Arerage Temperature Difference

Arerage Sample Temperature

Average Thermal Resistance $\mathrm{R}$

Arerage Thermal Conductance $C$ 9o minutes

$=15.48 \mathrm{Btu} /(\mathrm{h} \mathrm{sq}-\mathrm{ft})$

$=200.1 \operatorname{deg} F$

$=159.6$ deg $F$

$=40.5$ deg $F$

$=179.9 \operatorname{deg} F$

$=2.62 \mathrm{~h} \mathrm{sq}-\mathrm{ft} F / B \mathrm{H}$

$=0.382 \mathrm{Btu} /(\mathrm{h} \mathrm{sq}-\mathrm{ft} \mathrm{F})$

Calculations Based on Thickness Measured in Apparatus

Thickness.

Average Temperature Gradient

Apparent Thermal Conductivity $k$
$=2.020$ inch

$=20.06 \mathrm{deg} F /$ inch

$=0.772$ Btu-in/(h.sq-ft F)

operator: $\bigcirc$

Comments

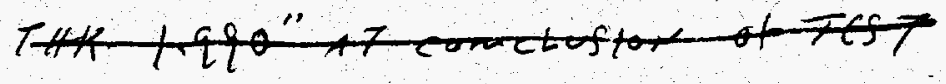

THK, 2.018 ATCOncLUS10n of TEST 
Req No: 950041

Date: $\quad 01-13-1995$

Steady-State Thermal Transmission Properties by Veans of the Heat Flow Weter per ASTM C $51 \mathrm{~S}$

Project No: 25? 155

Run ID: 257165

Sample Description: SAMPLE-L

Specimen Orientation: Horizontal Heat Meter Location: Cold Surface Instrument Used: Rapid-k \#2

Duration of Measurement:

Average Heat Flux

Average Hot plate Temperature Th

Average cold Plate Tomperature Tc

Average Temperature Difference

Arerage Sample Temperature

iverage Thermal Resistance $k$

Average Thermal conductance $\mathrm{C}$
131 minutes

$=14.75 \mathrm{Btu} /(\mathrm{h} \cdot \mathrm{sq}-\mathrm{ft})$

$=200.0 \operatorname{deg} \bar{F}$

$=159 . \mathrm{s} \mathrm{deg} F$

$=40.2 \operatorname{deg} F$

$=179.9 \operatorname{deg} F$

$=2.72 \mathrm{~h} s q-f t F / B t u$

$=0.367 \mathrm{Btu} /(\mathrm{h} \mathrm{sq}-\mathrm{ft} F)$

Calculations Based on Thickness Measured in Apparatus

Thickness

Average Temperature Gradient

Apparent Thermal Conductivityk
$=2.008$ inch

$=20.00 \mathrm{deg} F /$ inch

$=0.737$ Btu-in/(h sq-ft F)

Operator:

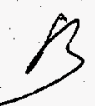

Comments 
$R \in q$ No: 950041

Date: 01-11-1995

Steady-State Thermal Transmission Properties

by Means of the Heat Flow Meter per ASTM C 518

Project No: 257165

Run ID: 257165

Sample Description: SAMPLEW

Specimen orientation: Horizontal Heat Meter Location: Cold Surface

Instrument Used: Rapid-k $\# 2$

Luration of Measurement:

Average Heat Flux

Average Hot Plate Temperature Th

Average cold Plate Temperature Tc

Average Temperature Difference

Average Sample Temperature

Average Thermal Resistance $R$

Average Thermal Conductance $\mathrm{C}$
71 minutes

$=15.75, \mathrm{Btu} /(\mathrm{h} \cdot \mathrm{sq}-\mathrm{ft})$

$=94.9 \operatorname{deg} F$

$=55.3$ deg $F$

$=39.6 \operatorname{deg} F$

$=75.1$ deg $F$

$=2.51$ h sq-ft F/Btu

$=0.398 \mathrm{Btu} /(\mathrm{h} \mathrm{sq}-\mathrm{ft} \mathrm{F}$ )

Calculations Based on Thickness Measured in Apparatus

Thickness

Average Temperature Gradient

Apparent Thermal Conductivity $\mathrm{k}$
$=2.017 \mathrm{inch}$

$=19.62 \mathrm{deg} \mathrm{F} / \mathrm{inch}$

$=0.803$ Btu-in/(h sq-ft F )

operator:

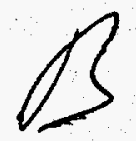

Comments 
Req No: 950041

Date: $\quad 01-10-1995$

Steady-State Thermal Transmission Properties

by Yans of the Heat Flow Meter per ASTM C 518

Project No: 257165

Sample Description: SAMPLE-W

Specimen Orientation: Horizontal

Instrument Used: Rapid-k\#2

Duration of Veasurement:

Arerage Heat Flux

Average Hot Plate Temperature Th

Arerage Cold Plate Temperature To

Average Temperature Difference

Average Sample Temperature

Average Thermal Resistance R

Average Thermal Conductance $C$
Run ID: 257165

\section{$s e=14.16$}

Heat Meter Location: Cold Surface 
Req No: 950041

Date: 01-13-1995

Steady-State Thermal Transmission properties by Means of the Heat Flow Meter per ASTM C 518

Project vio: 257165

Run ID: $25: 165$

Sample Description: SAMPLE-W

Specimen orientation: Horizontal Heat Meter Location: Cold Surface Instrument Used: Rapid-1 \#2

Duration of veasurement:

Average Heat $F !$ ux

Average Hot Plate Temperature Th

Average cold Plate Temperature To

Average Temperature Difference

Average Sample Temperature

Average Thermal Resistance $R$

Average Thermal conductance $C$
111. minutes

$=13.79 \mathrm{Btu} /(\mathrm{h} \mathrm{sq}-\mathrm{ft})$

$=200.0$ deg $F$

$=160.4 \operatorname{deg} F$

$=39.6$ deg $F$

$=180.2 \operatorname{deg~F}$

$=2.87 \mathrm{~h} \mathrm{sq-ft} \mathrm{F/Btu}$

$=0.348 \mathrm{Btu} /(\mathrm{h} \mathrm{sq}-\mathrm{ft} \mathrm{F})$

Calculations Based on Thickness Measured in Apparatus

Thickness

Average Temperature Gradient

Apparent Thermal Conductivity $k$
$=2.021$ inch

$=19.60$ deg F/inch

$=0.704 \mathrm{Btu}-\mathrm{in} /(\mathrm{h} \mathrm{sq}-\mathrm{ft} \mathrm{F})$

Operator:

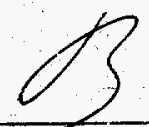

Comments 
Req No: 950041

Date: 01-13-1995

Steady-State Thermal Transmission properties by Means of the Heat Flow Meter per ASTM C 518

Project No: 257165

Run ID: 257165

Sample Description: SAMPLE-W

Specimen Orientation: Horizortal Heat Meter Location: Cold Surface Instrument Used: Rapid-k $\# 2$

Duration of Measurement:

Average Heat Flux

Average fot Plate Temperature. Th

Arerage Cold Plate Temperature To

Average Temperature Difference

Average Sample Temperature

Average Thermal Resistance $R$

Average Thermal Conductance $C$
$5+$ minutes

$=13.42 \mathrm{Btu} /(\mathrm{h} \mathrm{sq}-\mathrm{ft})$

$=200.1$ deg $F$

$=160.3$ deg $F$

$=39.7 \mathrm{deg} F$

$=180.2 \operatorname{deg} F$

$=2.96 \mathrm{~h} \mathrm{sq}-\mathrm{ft}$ FiBtu

$=0.338 \mathrm{Btu} /(\mathrm{h} \mathrm{sq}-\mathrm{ft} F)$

Calculations Based on Thickness Masured in Apparatus

Thickness

Average Temperature Gradient

Apparent Thermal Conductivity $\mathrm{k}$
$=2.021 \mathrm{inch}$

$=19.66 \mathrm{deg} F /$ inch

$=0.682$ Btu-in/(h sq-ft F)

Operator:

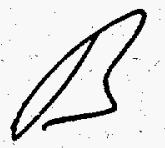

Comments 
Req No: 950041

Date: $01-17-1995$

Steady-state Thermal Transmission Properties by Means of the Heat Flow Meter per ASTM C 518

Project No: 257165

Run ID: $25-165$

Sample Description: SAMPLE-1-FLAT-AMB

Specimen orientation: Horizontal Heat Meter Location: Cold Surface Instrument Used: Rapid-k \#2

Duration of Measurement:

Average Heat Flux

Arerage Hot Plate Temperature Th

Average cold Plate Temperature TC

Average Temperature Difference

Average Sample Temperature

Average Thermal Resistance $R$

Average Thermal conductance $C$
175 minutes

$=29.54 \mathrm{Btu} /(\mathrm{h} \mathrm{sq}-\mathrm{ft})$

$=94.6 \operatorname{deg} \mathrm{F}$

$=54.7 \mathrm{deg} F$

$=39.9 \operatorname{deg} F$

$=74.6 \operatorname{deg} F$

$=1.35 \mathrm{~h} \mathrm{sq}-\mathrm{ft} F / \mathrm{Btu}$

$=0.739 \mathrm{Btu} /(\mathrm{h} s q-\mathrm{ft} \dot{\mathrm{F}})$

Calculations Based on Thickness Measured in Apparatus

Thickness

Average Temperature Gradient

Apparent Thermal Conductivity $k$
$=0.523 \mathrm{inch}$

$=76.36$ deg F/inch

$=0.387 \mathrm{Btu}-\mathrm{in} /(\mathrm{h}, \mathrm{sq}-\mathrm{ft} \mathrm{F})$

operator:

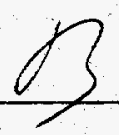

Comments 
Req No: 950041

Date: $\quad 01-17-1995$

Steady-State Thermal Transmission Froperties

by heans of the Heat Flow Meter per ASTM C 518

Project No: 257165

Run ID: 257165

Sample Description: SAMPLE-Z-FLAT-AMB

Specimen Orientation: Horizo tal Heat Meter Location: Cold Surface Instrument tsed: Rapid-k $\# 2$

Duration of Measurement:

Average Heat Flux

Average Hot Plate Temperature Th

Arerage cola plate Temperature To

Average Temperature Difference

Average Sample Temperature

Average Thermal Resistance $R$

Average Thermal Conductance $C$
52 minutes

$=30.17 \mathrm{Btu} /(\mathrm{h} \mathrm{sq}-\mathrm{ft})$

$=94.6 \operatorname{deg} F$

$=54.9$ deg $F$

$=39.7 \operatorname{deg} F$

$=-4.8$ deg $F$

$=1.32 \mathrm{~h} \mathrm{sq}-\mathrm{ft} F / \mathrm{Btu}$

$=0.759 \mathrm{Btu} /(\mathrm{h} \mathrm{sq}-\mathrm{ft} F)$

Calculations Based on Thickness Measured in Apparatus

Thickness

Average Temperature Gradient

Apparent Thermal Conductivity
$=0.515 \mathrm{inch}$

$=77.13 \operatorname{deg} F /$ inch

$=0.391 \mathrm{Btu}-\mathrm{in} /(\mathrm{h} \mathrm{sq}-\mathrm{ft} F)$

Cperator:

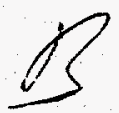


Req No: 950041

Date: 01-18-1995

Steady-State Thermal Transmission Properties by Heans of the Heat Flow Yeter per ASTM C 518

Project No: 257165 s.

Run ID: 257165

Sample Description: SAMPLE-1-2-FLAT-AMB

Specimen orientation: Horizontal Heat Neter Location: Cold Surface Instrument Used: Rapid-k \#2

Duration of Measurement:

$57 \cdot$ minutes

Average Heat Flux

Average Hot Plate Temperature Th

$=15.98 \mathrm{Btu} /(\mathrm{h} \mathrm{sq}-\mathrm{ft})$
$=94.8 \mathrm{deg} F$

Average cold Plate Temperature TC

$=55.0 \operatorname{deg} F$

Average Temperature Difference

$=39.8$ deg $F$

Average Sample Temperature

$=74.9 \operatorname{deg} \mathrm{F}$

Arerage Thermal Resistance $\pi$

$=2.49 \mathrm{~h} \mathrm{sq}-\mathrm{ft} / \mathrm{Ftu}$

Avarage Thermal Conductance. C

$=0.401 \mathrm{Btu} /(\mathrm{h} \mathrm{sq}-\mathrm{ft} F)$

Calculations Based on Thickness Measured in Apparatus

Thickness:

Average Temperature Gradient

Apparent Thermal conductivity $\mathrm{k}$
$=1.024 \mathrm{inch}$

$=38.39 \mathrm{deg} F /$ inch

$=0.411$ Btu-in/(h sq-ft F)

Operator:

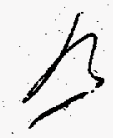

Comments 
Req No: 950041

Date: $01-18-1995$.

Steady-State Thermal Transmission properties

by veans of the Heat flow Veter per ASTM C 513

Project No: 25:165

Run ID: 257165

Sample Description: SAMPLE-3-FLAT-AMB

Specimen orientation: Horizontal Heat Meter Location: Cold Surface instrument Used: Rapid-k $\# 2$

Duration of Measurement:

inerage Heat Flux

Average Hot Plate Temperature Th

Average cold Plate Tomperature To

Average Temperature Difference

Average Sample Tomperature

Arrage Thermal Resistance $R$

Aierage Thermal Conductance $C$
56. minutes

$=30.26$ Btu/(h sq-ft)

$=94.9 \operatorname{deg} \mathrm{F}$

$=55.4 \operatorname{deg} F$

$=39.6, \operatorname{deg} F$

$=-5.1 \operatorname{deg} F$

$=1.31 \mathrm{~h} \mathrm{sq}-\mathrm{ft} \mathrm{F} / \mathrm{Btu}$

$=0.765 \mathrm{Btu} /(\mathrm{h} \mathrm{sq}-\mathrm{ft} F)$

Calculations Based on Thickness Measured in Apparatus

Thickness

Average Temperature Gradient

Apparent Thermal Conductivity $k$
$=0.511$ inch

$=77.41 \mathrm{deg} F /$ inch

$=0.391 \mathrm{Btu}-\mathrm{in} /(\mathrm{h} \mathrm{sq}-\mathrm{ft} F)$

operator:

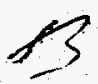

Comments 
Req No: 950041

Date: $\quad 01-18-1995$

Steady-State Thermal Transmission. Properties

ty Means of the Heat Flow Meter per ASTM C 518

Project No: 257165

Run ID: 257165

Sample Description: SAMPLE-1-2-3-FLAT AMB

Specimen Orientation: Horizontal Heat Meter Location: Cold Surface instrument Used: Rapid-k\#2

Duration of Measurement:

Average Heat Flux

Average Hot Plate Temperature Th

Average Cold Plate Temperature Tc

Average Temperature Difference

Average Sample Temperature

Average Thermal Resistance $R$

Average Thermal Conductance $C$
54 minutes

$=11.04 \mathrm{Btu} /(\mathrm{h} \mathrm{sq}-\mathrm{ft})$

$=34.9$ deg $F$

$=54.8$ deg $\mathrm{F}$

$=40.1 \operatorname{deg} \mathrm{F}$

$=74.8 \mathrm{deg} F$

$=3.63 \mathrm{~h} \mathrm{sq}-\mathrm{ft} \mathrm{F} / \mathrm{Btu}$

$=0.276 \mathrm{Btu} /(\mathrm{h} \mathrm{sq}-\mathrm{ft} \mathrm{F})$

Calculations Based on Thickness Measured in Apparatus

Thickness

Average Temperature Gradient

Apparent Thermal Conductivity $k$
$=1.547$ inch

$=25.90 \mathrm{deg} F /$ inch

$=0.426 \mathrm{Btu}-\mathrm{in} / \mathrm{h}$ sq-ft

operator:

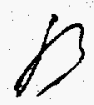

Comments 
Req No: 950041

Date: $01-18-1995$

Steady-State Thernal Transmission Properties by deans of the lleat Flow teter per isThe $51 \mathrm{~S}$

Project No: 257165

Fun ID: 25?165

Sample Description: SAMPLE-4-FLAT-AMB

Specinen orientation: Horizontal Heat Meter Location: Cold Surface instiment Used: Rapid-k $\# 2$

Duration of yeasurement:

60. minutes

Arerage Heat Flux

$=28.76 \mathrm{Btu} /(\mathrm{h} \mathrm{sq}-\mathrm{ft})$

Average Hot Plate Temperature Th

$=94.8 \operatorname{deg} F$

Average Cold Fiate Temperature To

$=55.0$ deg $T$

Average Temperature Difference

$=39.5$ deg F

Average Sample Temperature

$=-4.9 \mathrm{deg} F$

Arerage Thermal Resistaned $R$

$=1.38 \mathrm{~h} \mathrm{sq-ft}$ F/Btu

AVerage Thermal Conductance $C$

$=0.723$ Btu/(h sq-ft F)

Calculations Based on Thickness Measured in Apparatus

Thiskness

Arerage Temperature Gradient

ipparent Thermal Conductivity $\mathrm{k}$
$=0.527$ inch

$=-5.47$ deg F/inch

$=0.381 \mathrm{Btu}-\mathrm{in} /(\mathrm{h} s \mathrm{q}-\mathrm{ft} \mathrm{F})$

Oparator:

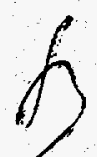

Cominents 
Req No: 950041

Date: $01-18-1995$

Steady-State Thermal Transmission Properties

by Heans of the Heat Flow Meter per ASTM C Sis

Projed ino: 257165

Run ID: 257165

Sample Description: SAMPLE-1-2-3-4-PLAT-AMB

Specimen Orientation: Horizontal Heat Meter Location: Cold Surface Instrument Used: Rapid-k \#2

Duration of Measurement:

Average Heat Flü

Average Hot Plate Temperature Th

Arerage Cold Plate Temperature To

Average Temperature Difference

Arerage Sampie Temperature

Average Thermal Resistance $R$

Averaze Thermal Conductance $C$
86 minutes

$=7.93$ Btu/(h sq-ft)

$=95.1 \mathrm{deg} F$

$=55.7 \operatorname{deg} F$

$=39.4 \operatorname{deg} \mathrm{F}$

$=75.4 \mathrm{deg} F$

$=4.96 \mathrm{~h} \mathrm{sq-ft} \mathrm{F/Btli}$

$=0.201 \mathrm{Btu} /(\mathrm{h} \mathrm{sq}-\mathrm{ft} F)$

Calculations Based on Thickness Measured in Apparatus

Thickness

Average Temperature Gradient

Apparent Thermal Conductivity $k$
$=2.058$ inch

$=19.12 \mathrm{deg}$ F/inch

$=0.415 \mathrm{Btu}-\mathrm{in} / \mathrm{h} \mathrm{sq}-\mathrm{ft}$

Operator:

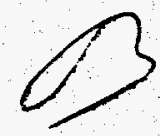

Comments 
Req No: 950041

Date: 01-13-1995

Steady-state Thermal Transmission Properties by Means of the Heat Flow Meter per isTM C 518

Froject No: $25-165$

Run ID: 257165

Sample Description: SAMPLE-1 FLAT

Specimen Orientation: Horizontal Heat Meter Location: Cold Surface instrument Used: Rapid-k \#2

Duration of Measurement:

Arerage heat Flua

Average Hot Flate Temperature Th

Average Cold Plate Temperature Tc

$\therefore$ rerage Temperature Difference

Average Sample Temperature

Average Thermal Resistance $R$

Arerage Thermal conductance $C$
53 minutes

$=35.28$ Btu/(h sq-ft $)$

$=200.0^{\circ} \cdot \operatorname{deg} F$

$=160.4 \operatorname{deg} F$

$=39.6$ des $F$

$=180.2$ deg $F$

$=1.12 \mathrm{~h} \mathrm{sq}-\mathrm{ft} / \mathrm{F} / \mathrm{Btu}$

$=0.891$ Btu/(h sq-ft F).

Calculations Based on Thickness Measured in Apparatus

Thickness

Average Temperature Gradient

Apparent Thermal Conductivity $k$
$=0.527$ inch

$=75.13 \mathrm{deg} F /$ inch

$=0.470$ Btu-in/(h sq-ft $F)$

Operator:

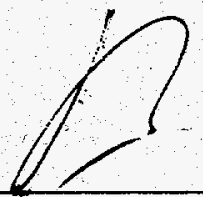

Comments 
Req No: $9500+1$

Date: $\quad 01-17-1995$

Steady-state Thermal Transmission Properties
by Means of the Heat Flow Hetei per ASTM C $\$ 18$

Project No: 257165

Run ID: 257165

Sample Description: SAMPLE-2-FLAT

Specimen Crientation: Horizontal Heat Meter Location: Cold Surface Instrument Lsed: Rapid-k $\# 2$

Duration of Measurement:

Average Heat Flux

Average Hot Plate Temperature Th

Average Cold Plate Temperature TC

Average Temperature Difference

Average Sample Temperature

Arerage Thermal Resistance $R$

Average Thermal conductance $c$
56 minutes

$=34.67 \quad \mathrm{Btu} /(\mathrm{h}$ sq-ft $)$

$=200.0 \operatorname{deg} F$

$=160.1 \operatorname{deg} F$

$=39.9$ deg $F$

$=180.1 \operatorname{deg} F$

$=1.15 \mathrm{~h} \mathrm{sq}-\mathrm{ft} / \mathrm{F} / \mathrm{Btu}$

$=0.369$ Btu/(h sq-ft F)

Calculations Based on Thickness Measured in Apparatus

Thickness

Average Femperature Gradient

Apparent Thermal Conductivity $k$
$=0.529 \dot{\mathrm{inch}}$

$=75.42 \mathrm{deg} F /$ inch

$=0.460 \mathrm{Btu}-\mathrm{in} /(\mathrm{h} s q-f t F)$

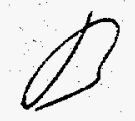

operator:

Comments 
Req No: $9500+1$

Date: $01-13-1995$

Steady-State Thermal Transmission Properties

by Heans of the Heat Flow Moter per AsTh C 518

Project ro: $2=-155$

Run ID: 25:165

Sample Deseription: SAMELE-1+2-FLAT

Specimen Orientation: Horizontal Heat Meter Location: Cold Surface Instrunert lsed: Rapid-k

Duration of Measurement:

Arerage Heat Flux

Aterag Hot Flate Temperatire. Th

Average cold plate Temperature To

iverage Temperature Difference

Areraze sample remperature

Averaga Thermal Rssistance $\mathrm{k}$

Nerage Thermal conductance $C$
90 minutes

$=16.38 \mathrm{Btu} /(\mathrm{h} \mathrm{sq}-\mathrm{ft})$

$=200.2 \operatorname{deg} F$

$=159.6 \mathrm{deg} F$

$=40.6$ deg $\mathrm{F}$

$=179.9 \operatorname{deg} \mathrm{F}$

$=2.48 \mathrm{~h} \mathrm{sq}-\mathrm{ft} / \mathrm{F} / \mathrm{Btu}$

$=0.403 \mathrm{Btu} /(\mathrm{h} \mathrm{sq}-\mathrm{ft} F)$

Calculations Based on Thickness Masured in Apparatus

Thickness

Average Temperature Gradient

Apparent Thermal Conductivity $k$
$=1.041 \mathrm{inch}$

$=39.02 \mathrm{deg} F /$ inch

$=0.420 \mathrm{Btu}-\mathrm{in} /(\mathrm{h} \mathrm{sq}-\mathrm{ft} \mathrm{F})$

Operator:

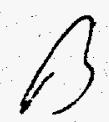

Comments 
Req No: 950041

Date: $\quad 01-16-1995$

Steady-State Thermal Transmission Properties

by Means of the Heat Flow Meter per ASTM C 518

Froject No: 257165

Run ID: 257165

Sample Description: SAMPLE-3-FLAT

Specimen Orientation: Horizontal Heat Meter Location: Cold Surface Instrument Used: Kapid-k \#2

Duration of Measurement:

Average Heat Flux

Average Hot Plate Temperature Th

Average cold Plate Temperature Tc

Average Temperature Difference

Average Sample Temperature

Average Thermal Resistance $R$

Aierage Thermal Conductance $C$
111 minutes

$=34.06 \mathrm{Btu} /(\mathrm{h} \mathrm{sq}-\mathrm{ft})$

$=200.1$ deg $F$

$=160.0 \quad \operatorname{deg} F$

$=40.1 \operatorname{deg} F$

$=150.1 \operatorname{deg} F$

$=1.1 \mathrm{~s} h \mathrm{sq}-\mathrm{ft} F / \mathrm{Btu}$

$=0.850 \mathrm{Btu} /\left(\mathrm{h}^{\mathrm{s}} \mathrm{sq}-\mathrm{ft} \mathrm{F}\right)$

Calculations Based on Thickness Measured in Apparatus

Thickness

Average Temperature Gradient

Apparent Thermal Conductivity $k$
$=0.529 \mathrm{inch}$

$=75.76 \mathrm{deg} F /$ inch

$=0.450$ Btu-in/(h sq-ft F)

operator:

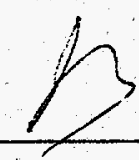

Comments 

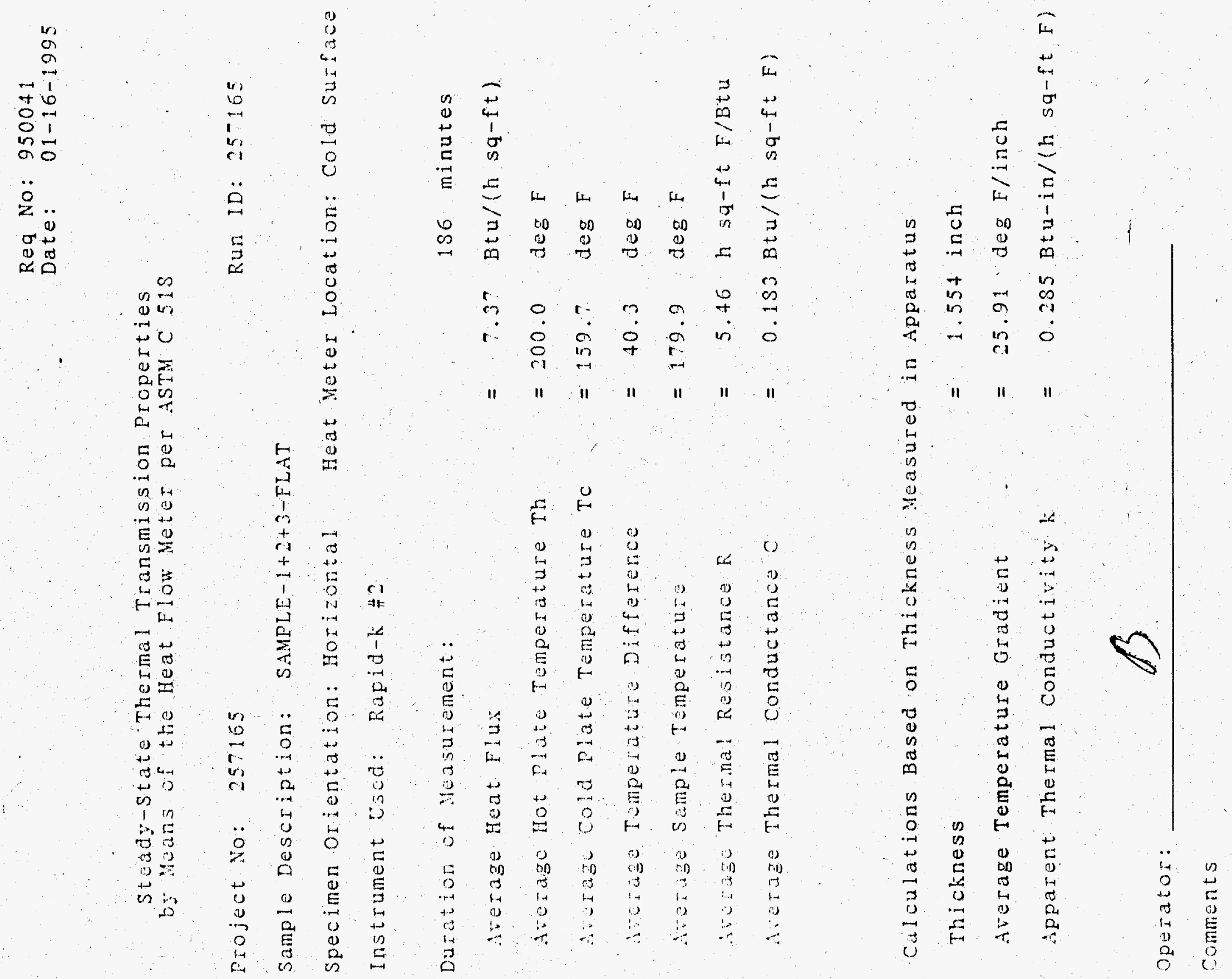
Req No: $950041^{\circ}$

Date: $\quad 01-17-1995$

Stedy-state Thermal Transmission Properties Heans of the Heat Flow Meter per ASTM C 518

Brojuz: No: 2: $25-165$

Run ID: 257165

Sanple Description: SAMPLE-4-FLAT

Specimer Orientation: Horizontal Heat Meter Location: Cold Surface Injtument Used: Rapid-k

Duration of Measurement:

Arerage Hat Fluk

Surage rot liate Temperature Th

Arago Cold Elate Temperature To

Arate Temperature Diffarance

Average Sample Temperature

$\therefore$ arge Thermal Resistance $R$

sterage Thermal conductance $C$
56 minutes

$=33.78 \mathrm{Btu} /(\mathrm{h} \mathrm{sq}-\mathrm{ft})$

$=200.2 \operatorname{deg} F$

$=160.4$ deg $F$

$=39.8$ deg $F$

$=130.3 \operatorname{deg} F$

$=1.1 s$ h sq-ft F/Btu

$=0.849 \mathrm{Btu} /(\mathrm{h} s \mathrm{q}-\mathrm{ft} F)$

Calculations Based on Thickness Measured in Apparatus

Thickness

Average Temperature Gradient Apparent Thermal Conductivity $k$
$=0.528$ inch

$=75.32 \mathrm{deg} F /$ inch

$=0.449$ Btu-in/(h sq-ft

operator:

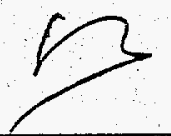


Req No: 950041

Date: $\quad 01-16-1995$

Steady-State Thermal Transmission Properties by Heans of the Heat Flow Meter per ASTM C 518

Project No: 25.7165

Run ID: 257165

Sample Description: SAMPLE-1+2+3+4-FLAT

Specimen orientation: Horizontal Heat Meter Location: Cold Surface Instrument Used: Rapid-k \#2

Duration of Measurement:

Average Heat Flux

Average Hot Plate Temperature Th

Average cold Plate Temperature TC

Average Temperature Difference

Averaze Sample Temperature

Average Thermal Resistance $R$

Alerage Thermal Conductance $\mathrm{C}$
185 minutes

$=3.21 \mathrm{Btu} /(\mathrm{h} \mathrm{sq}-\mathrm{ft})$

$=200.2$ deg $F$

$=160.0 \cdot \operatorname{deg} F$

$=40.2 \operatorname{deg} F$

$=180.1 \cdot \operatorname{deg} F$

$=12.52 \mathrm{~h} \mathrm{sq}-\mathrm{ft} \mathrm{F} / \mathrm{Btu}$

$=0.080 \mathrm{Btu} /(\mathrm{h} \mathrm{sq}-\mathrm{ft} \mathrm{F})$

Calculations Based on Thickness Measured in Apparatus

Thickness

Average Temperature Gradient

Apparent Thermal Conductivity $k$
$=2.064$ inch

$=19.49 \mathrm{deg} F /$ inch

$=0.165$ Btu-in/(h sq-ft

Operátor:

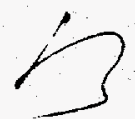

comments 
Req No:

Date: $\quad 02-20-1995$

Steady-State Thermal Transmission Properties

by Means of the Heat Flow Meter per ASTM C 518

Project No: 950245

RUn ID: GERRY

Sample Description: FIBER-BOARD-GERRY

Specimen Orientation: Horizontal Heat Meter Location: Cold Surface Instrument Used: Rapid-k \#2

Duration of Measurement:

Average Heat Flux

Average Hot Plate Temperature Th

Average Cold Plate Temperature Tc

Average Temperature Difference

Average Sample Temperature

Average Thermal Resistance $R$

Average Thermal Conductance $\mathrm{C}$
174 minutes

$=8.37$ Btu/(h sq-ft $)$

$=200.0 \operatorname{deg} \mathrm{F}$

$=160.0 \quad \operatorname{deg} F$

$=40.0$ deg $F$

$=180.0 \operatorname{deg} F$

$=4.78 \mathrm{~h} \mathrm{sq}-\mathrm{ft} F /$ Btu

$=0.209$ Btu/(h sq-ft F $)$

Calculations Based on Thickness Measured in Apparatus

Thickness

Average Temperature Gradient

Apparent Thermal Conductivity $k$

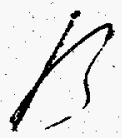

$=2.055$ inch

$=19.46 \mathrm{deg} F /$ inch

$=0.430$ Btu-in $/(h$ sq-ft F $)$

Operator:

Comments 
Req No:

Date: $\quad 02-20-1995$

Steady-State Thermal Transmission Properties

by Means of the Heat Flow Meter per ASTM C 518

Project No: 950245

Run ID: GERRY

Sample Description: FIBER-BOARD-GERRY

Specimen Orientation: Horizontal Heat Meter Location: Cold Surface Instrument Used: Rapid-k \#2

Duration of Measurement:

Average Heat Flux

Average Hot Plate Temperature Th

Average cold Plate Temperature Tc

Average Temperature Difference

Average Sample Temperature

Average Thermal Resistance $\mathrm{R}$

Average Thermal Conductance $\mathrm{C}$
53 minutes

$=8.59 \cdot \mathrm{Btu} /(\mathrm{h} \mathrm{sq}-\mathrm{ft})$

$=199.9 \operatorname{deg} \mathrm{F}$

$=160.0 \operatorname{deg} F$

$=39.9 \operatorname{deg} \mathrm{F}$

$=179.9 \operatorname{deg} \mathrm{F}$

$=4.64 \mathrm{~h} \mathrm{sq}-\mathrm{ft} \mathrm{F} / \mathrm{Btu}$

$=0.215 \mathrm{Btu} /(\mathrm{h} \mathrm{sq}-\mathrm{ft} \mathrm{F})$

Calculations Based on Thickness Measured in Apparatus

Thickness

Average Temperature Gradient

Apparent Thermal Conductivity $k$
$=2.055 \mathrm{inch}$

$=19.42 \mathrm{deg}$ F/inch

$=0.442 \mathrm{Btu}-\mathrm{in} /(\mathrm{h} \mathrm{sq}-\mathrm{ft} \mathrm{F})$

Operator:

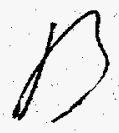

Comments 
Req No:

Date: $\quad 02-20-1995$

Steady-State Thermal Transmission Properties
by Means of the Heat Flow Meter per ASTM C 518

Project No: 950245

RUn ID: GERRY

Sample Description: FIBER-BOARD-GERRY

Specimen Orientation: Horizontal Heat Meter Location: Cold Surface Instrument Used: Rapid-k \#2

Duration of Measurement:

Average Heat Flux

Average Hot Plate Temperature Th

Average cold Plate Temperature TC

Average Temperature Difference

Average Sample Temperature

Average. Thermal Res istance $R$

Average Thermal Conductance $C$
52 minutes

$=8.63 \mathrm{Btu} /(\mathrm{h} \mathrm{sq}-\mathrm{ft})$

$=200.0 \quad \operatorname{deg} F$

$=160.1 \operatorname{deg} \mathrm{F}$

$=39.9 \mathrm{deg} F$

$=180.0 \quad \operatorname{deg} \mathrm{F}$

$=4.63 \mathrm{~h} s q-f \mathrm{t} / \mathrm{Btu}$

$=0.216 \mathrm{Btu} /(\mathrm{h} \mathrm{sq}-\mathrm{ft} F)$

Calculations Based on Thickness Measured in Apparatus

Thickness

Average Temperature Gradient

Apparent Thermal Conductivity $k$
$=2.055 \mathrm{inch}$

$=19.42 \mathrm{deg} F /$ inch

$=0.444$ Btu-in/(h sq-ft F )

Operator:

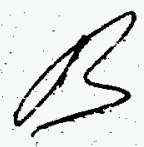

Comments 
Req No:

Date: $\quad 02-20-1995$

Steady-state Thermal Transmission Properties by Means of the Heat Flow Meter per ASTM C 518

Project No: 950245

Run ID: GERRY

Sample Description: FIBER-BOARD-GERRY

Specimen Orientation: Horizontal Heat Meter Location: Cold Surface Instrument Used: Rapid-k \#2

Duration of Measurement:

Average Heat Flux

Average Hot Plate Temperature Th

Average Cold Plate Temperature TC

Average Temperature Difference

Average Sample Temperature

Average Thermal Resistance $R$

Average Thermal Conductance $\mathrm{C}$
53 minutes

E $\quad 8.60 \mathrm{Btu} /(\mathrm{h} \mathrm{sq}-\mathrm{ft})$

$=200.0 \operatorname{deg} \mathrm{F}$

$=160.0 \operatorname{deg} F$

$=40.0 \operatorname{deg} F$

$=180.0 \operatorname{deg} F$

$=4.64 \mathrm{~h} \mathrm{sq-ft}$ F/Btu

$=0.215 \mathrm{Btu} /(\mathrm{h} \mathrm{sq}-\mathrm{ft} \mathrm{F})$

Calculations Based on Thickness Measured in Apparatus

Thickness

Average Temperature Gradient

Apparent Thermal Conductivity k
$=2.055 \mathrm{inch}$

$=19.44 \mathrm{deg} F /$ inch

$=0.443 \mathrm{Btu}-\mathrm{in} /(\mathrm{h} \mathrm{sq}-\mathrm{ft} \mathrm{F})$

Operator:

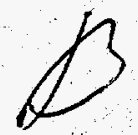

Comments 
Req No:

Date: $\quad 02-20-1995$

Steady-state Thermal Transmission Properties by Means of the Heat Flow Meter per ASTM C 518

Project No: 950245

Run ID: GERRY

Sample Description: FIBER-BOARD-GERRY

Specimen Orientation: Horizontal Heat Meter Location: Cold Surface Instrument Used: Rapid-k \#2

Duration of Measurement:

Average Heat Flux

Average Hot Plate Temperature Th

Average Cold Plate Temperature Tc

Average Temperature Difference

Average Sample Temperature

Average Thermal Resistance $R$

Average Thermal Conductance $\mathrm{C}$
54 minutes

$=8.57$ Btu/(h sq-ft $)$

$=199.9 \operatorname{deg} F$

$=160.0 \operatorname{deg} F$

$=40.0 \operatorname{deg} F$

$=180.0 \operatorname{deg} F$

$=4.66 \mathrm{~h}$ sq-ft $\mathrm{F} / \mathrm{Btu}$

$=0.215 \mathrm{Btu} /(\mathrm{h} \mathrm{sq}-\mathrm{ft} F)$

Calculations Based on Thickness Measured in Apparatus

Thickness

Average Temperature Gradient

Apparent Thermal Conductivity $k$
$=2.055 \mathrm{inch}$

$=19.45 \mathrm{deg} / \mathrm{F} / \mathrm{nch}$

$=0.441 \mathrm{Btu}-\mathrm{in} /(\mathrm{h} \mathrm{sq}-\mathrm{ft} \mathrm{F})$

Operator:

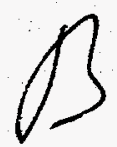

Comments 
Req No:

Date: $\quad 02-20-1995$

Steady-state Thermal Transmission Properties

by Means of the Heat Flow Meter per ASTM C 518

Project No: 950245

Run ID: GERRY

Sample Description: FIBER-BOARD-GERRY

Specimen Orientation: Horizontal Heat Meter Location: Cold Surface Instrument Used: Rapid-k \#2

Duration of Measurement:

Average Heat Flux

Average Hot Plate Temperature Th

Average cold Plate Temperature Tc

Average Temperature Difference.

Average Sample Temperature

Average Thermal Resistance $R$

Average Thermal Conductance C
52 minutes

$=8.58 \mathrm{Btu} /(\mathrm{h} \mathrm{sq}-\mathrm{ft})$

$=199.9 \operatorname{deg} \mathrm{F}$

$=159.9 \operatorname{deg} \mathrm{F}$

$=40.0$ deg $\mathrm{F}$

$=179.9 \operatorname{deg} \mathrm{F}$

$=4.66 \mathrm{~h} \mathrm{sq}-\mathrm{ft} \mathrm{F} / \mathrm{Btu}$

$=0.215 \mathrm{Btu} /(\mathrm{h} \mathrm{sq}-\mathrm{ft} \mathrm{F})$

Calculations Based on Thickness Measured in Apparatus

Thickness

Average Temperature Gradient

Apparent Thermal Conductivity $k$
$=2.055 \mathrm{inch}$

$=19.45 \mathrm{deg} / \mathrm{F} / \mathrm{inch}$

$=0.441$ Btu-in/(h sq-ft F )

Operator:

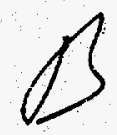

Comments 
Req No:

Date:

$02-22-1995$

Steady-state Thermal Transmission Properties

by Means of the Heat Flow Meter per ASTM C 518

Project No: 277165

RUN ID: GERRY

Sample Description: FIBERBOARD-GERRY-AMB - 75

Specimen Orientation: Horizontal Heat Meter Location: Cold Surface Instrument Used: Rapid-k \#2

Duration of Measurement:

Average Heat Flux

Average Hot Plate Temperature Th

Average Cold Plate Temperature TC

Average Temperature Difference

Average Sample Temperature

Average Thermal Resistance $R$

Average Thermal Conductance $C$
94 minutes

$=7.67 \mathrm{Btu} /(\mathrm{h} \mathrm{sq}-\mathrm{ft})$

$=94.7 \mathrm{deg} F$

$=55.1 \mathrm{deg} F$

$=39.6 \mathrm{deg} F$

$=74.9 \operatorname{deg} \mathrm{F}$

$=5.16 \mathrm{~h} \mathrm{sq}-\mathrm{ft}$ F/Btu

$=0.194 \mathrm{Btu} /(\mathrm{h} \mathrm{sq}-\mathrm{ft} F)$

Calculations Based on Thickness Measured in Apparatus

Thickness

Average Temperature Gradient

Apparent Thermal Conductivity $k$
$=2.038$ inch

$=19.43 \mathrm{deg} / \mathrm{F} / \mathrm{inch}$

$=0.395 \mathrm{Btu}-\mathrm{in} /(\mathrm{h}$ sq-ft F $)$

Operator:

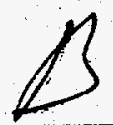

Comments 
Req No:

Date :

$02-22-1995$

Steady-State Thermal Transmission Properties

by Means of the Heat Flow Meter per ASTM C 518

Project No: 277165

Run ID: GERRY

Sample Description: FIBERBOARD-GERRY-AMB -75

Specimen Orientation: Horizontal Heat Meter Location: Cold Surface Instrument Used: Rapid-k \#2

Duration of Measurement:

Average Heat Flux

Average Hot Plate Temperature Th

Average Cold Plate Temperature Tc

Average Temperature Difference

Average Sample Temperature

Average Thermal Resistance $R$

Average Thermal Conductance $\mathrm{C}$
52 minutes

$=7.79$ Btu/(h sq-ft $)$

$=94.7 \operatorname{deg} F$

$=55.0 \mathrm{deg} F$

$=39.6 \operatorname{deg~F}$

$=74.9 \operatorname{deg} \mathrm{F}$

$=5.09 \mathrm{~h} \mathrm{sq}-\mathrm{ft}$ F/Btu

$=0.196 \mathrm{Btu} /(\mathrm{h} \mathrm{sq}-\mathrm{ft} F)$

Calculations Based on Thickness Measured in Apparatus

Thickness

Average Temperature Gradient

Apparent Thermal Conductivity $k$
$=2.038$ inch

$=19.45 \mathrm{deg} / \mathrm{F} / \mathrm{inch}$

$=0.400 \mathrm{Btu}-\mathrm{in} /(\mathrm{h} s q-\mathrm{ft} F)$

Operator:

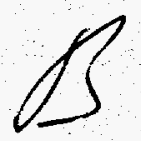

Comments 
Req No:

Date:

$02-22-1995$

Steady-State Thermal Transmission Properties

by Means of the Heat Flow Meter per ASTM C 518

Project No: 277165

Run ID: GERRY

Sample Description: FIBERBOARD-GERRY-AMB. -75

Specimen Orientation: Horizontal Heat Meter Location: Cold Surface Instrument Used: Rapid-k \#2

Duration of Measurement:

Average Heat Flux

Average Hot Plate Temperature Th

Average Cold Plate Temperature Tc

Average Temperature Difference

Average Sample Temperature

Average Thermal Resistance $R$

Average Thermal Conductance $\mathrm{C}$
55 minutes

$=7.77 \mathrm{Btu} /(\mathrm{h} \mathrm{sq}-\mathrm{ft})$

$=94.7 \operatorname{deg} \mathrm{F}$

$=55.0 \operatorname{deg} \mathrm{F}$

$=39.7 \operatorname{deg} F$

$=74.9$ deg $F$

$=5.11 \mathrm{~h}$ sq-ft F/Btu

$=0.196 \mathrm{Btu} /(\mathrm{h} \mathrm{sq}-\mathrm{ft} F)$

Calculations Based on Thickness Measured in Apparatus

Thickness

Average Temperature Gradient

Apparent Thermal Conductivity $k$
$=2.038 \mathrm{inch}$

$=19.47 \mathrm{deg} F /$ inch

$=0.399$ Btu-in/(h sq-ft F)

Operator:

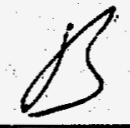

Comments 
Req No:

Date: $\quad 02-22-1995$

Steady-State Thermal Transmission Properties

by Means of the Heat Flow Meter per ASTM C 518

Project No: 277165

Run ID: GERRY

Sample Description: FIBERBOARD-GERRY-AMB . -75

Specimen Orientation: Horizontal Heat Meter Location: Cold Surface Instrument Used: Rapid-k \#2

Duration of Measurement:

Average Heat Flux

Average Hot Plate Temperature Th

Average Cold Plate Temperature TC

Average Temperature Difference

Average Sample Temperature

Average Thermal Resistance $R$

Average Thermal Conductance $\mathrm{C}$
56 minutes

$=7.74 \mathrm{Btu} /(\mathrm{h}$ sq-ft $)$

$=94.7 \operatorname{deg} \mathrm{F}$

$=55.0 \operatorname{deg} \mathbf{F}$

$=39.7 \mathrm{deg} F$

$=74.9$ deg $F$

$=5.13 \mathrm{~h} \mathrm{sq}-\mathrm{ft} \mathrm{F} / \mathrm{Btu}$

$=0.195 \mathrm{Btu} /(\mathrm{h}$ sq-ft F $)$

Calculations Based on Thickness Measured in Apparatus

Thickness

Average Temperature Gradient

Apparent Thermal Conductivity k

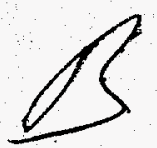

Operator:
$=2.038$ inch
$=19.48 \mathrm{deg}$ F/inch
$=0.398 \mathrm{Btu}-\mathrm{in} /(\mathrm{h} \mathrm{sq}-\mathrm{ft} \mathrm{F})$

Comments 
Req No:

Date:

$02-22-1995$

Steady-State Thermal Transmission Properties

by Means of the Heat Flow Meter per ASTM C 518

Project No: 277165

Run ID: GERRY

Sample Description: FIBERBOARD-GERRY-AMB, -75

Specimen Orientation: Horizontal Heat Meter Location: Cold Surface Instrument Used: Rapid-k \#2

Duration of Measurement:

Average Heat Flux

Average Hot Plate Temperature Th

Average Cold Plate Temperature Tc

Average Temperature Difference

Average Sample Temperature

Average Thermal Resistance $R$

Average Thermal Conductance $\mathrm{C}$
52 minutes

$=7.74$ Btu/(h sq-ft $)$

$=94.8 \operatorname{deg} F$

$=55.1 \quad \operatorname{deg} F$

$=39.7$ deg $F$

$=75.0$ deg $F$

$=5.13 \mathrm{~h} \mathrm{sq-ft} \mathrm{F/Btu}$

$=0.195 \mathrm{Btu} /(\mathrm{h} \mathrm{sq}-\mathrm{ft} F)$

Calculations Based on Thickness Measured in Apparatus

Thickness

Average Temperature Gradient

Apparent Thermal Conductivity $k$
$=2.038 \mathrm{inch}$

$=19.48 \mathrm{deg} / \mathrm{F} / \mathrm{inch}$

$=0.397$ Btu-in/(h sq-ft F)

Operator:

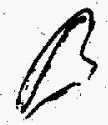

Cómments 
Req No:

Date: $02-22-1995$

Steady-state Thermal Transmission Properties

by Means of the Heat Flow Meter per ASTM C 518

Project No: 277165

Run ID: GERRY

Sample Description: FIBERBOARD-GERRY-AMB. -75

Specimen Orientation: Horizontal Heat Meter Location: Cold Surface Instrument Used: Rapid-k \#2

Duration of Measurement:

Average Heat Flux

Average Hot Plate Temperature $T h$

Average Cold Plate Temperature TC

Average Temperature Difference

Average Sample Temperature

Average Thermal Resistance $R$

Average Thermal Conductance $\mathrm{C}$
53 minutes

$=7.75 \mathrm{Btu} /(\mathrm{h} \mathrm{sq}-\mathrm{ft})$

$=94.7 \operatorname{deg} \mathrm{F}$

$=55.0 \quad \operatorname{deg} F$

$=39.8 \operatorname{deg} F$

$=74.9 \operatorname{deg} F$

$=5.13 \mathrm{~h} \mathrm{sq-ft}$ F/Btu

$=0.195 \mathrm{Btu} /(\mathrm{h} \mathrm{sq}-\mathrm{ft} F)$

Calculations Based on Thickness Measured in Apparatus

Thickness

Average Temperature Gradient

Apparent Thermal Conductivity $k$
$=2.038 \mathrm{inch}$

$=19.51 \mathrm{deg} / \mathrm{F} / \mathrm{nch}$

$=0.397 \mathrm{Btu}-\mathrm{in} /(\mathrm{h} \mathrm{sq}-\mathrm{ft} \mathrm{F})$

Operator:

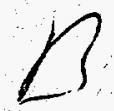

Comment s 
Req No:

Date:

Steady-State Thermal Transmission Properties by Means of the Heat Flow Meter per ASTM C 518

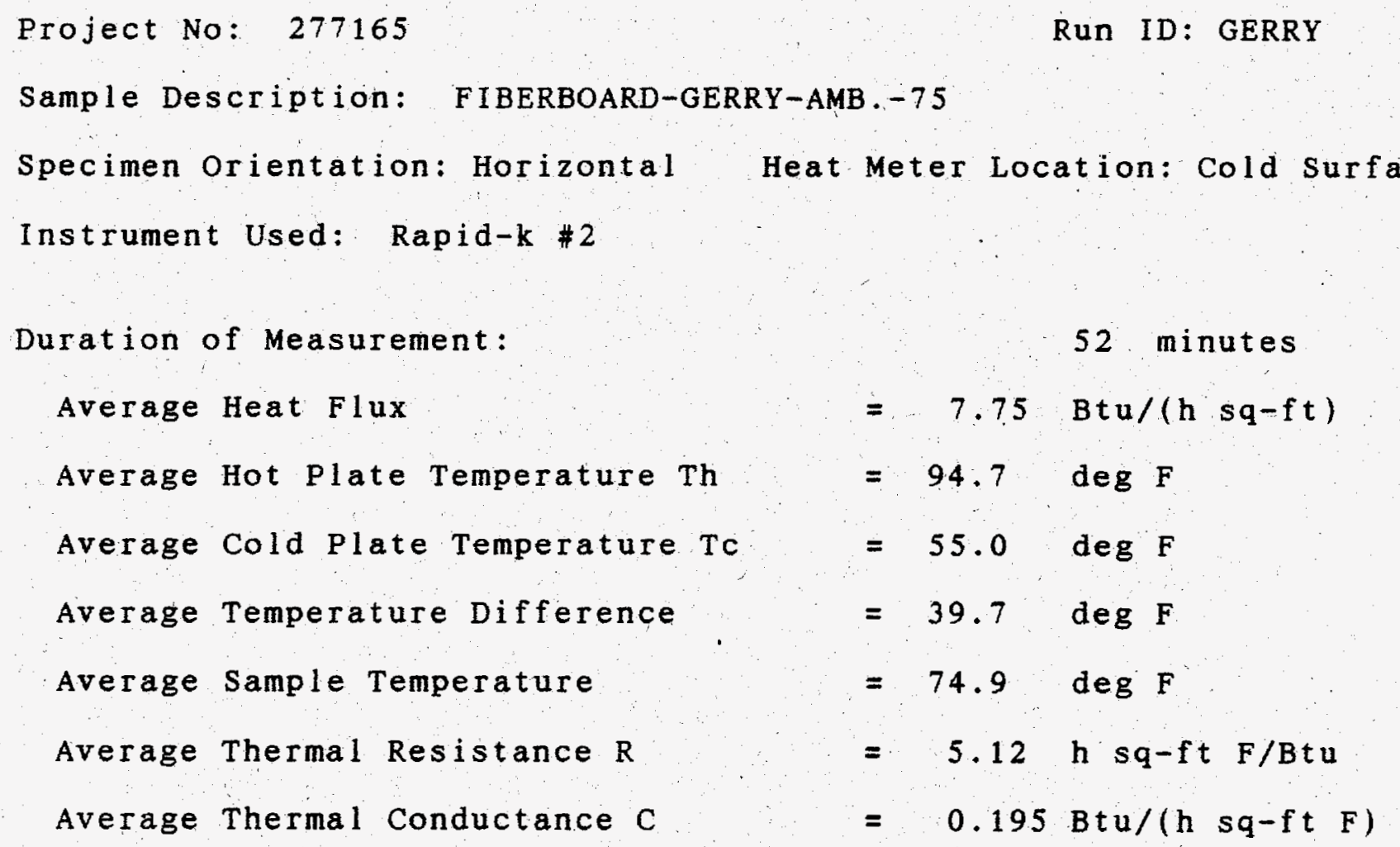

Calculations Based on Thickness Measured in Apparatus

Thickness

Average Temperature Gradient

Apparent Thermal Conductivity $k$
$=2.038 \mathrm{inch}$

$=19.49 \mathrm{deg}$ F/inch

$=$

$0.398 \mathrm{Btu}-\mathrm{in} /(\mathrm{h} \mathrm{sq-ft} \mathrm{F}$ )

Operator:

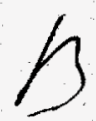

Comments 